\title{
Conf-95)03/6-1
}

Paper to be presented at the 7th Portuguese Conference on Artificial Intelligence, Funchal, Madeira Island, Portugal, October 3-6, 1995.

RECFIVFD

JAN $30 \quad 1995$

$0>1$,

Systematic Construction of Qualitative Physics-Based Rules for Process Diagnostics*

by

J. Reifman

Reactor Analysis Division

Argonne National Laboratory

9700 South Cass Avenue

Argonne, Il 60439

T. Y. C. Wei

Reactor Engineering Division

Argonne National Laboratory

9700 South Cass Avenue

Argonne, IL 60439

The submitted manuscript has been authored
by a contractor of the U.S. Government
under contract No. W-31-109-ENG-38.
Accordingly, the U. S. Government retains a
nonexclusive, royalty-free license to publish
or reproduce the published form of this
contribution, or allow others to do so, for
$U . S$. Government purposes.

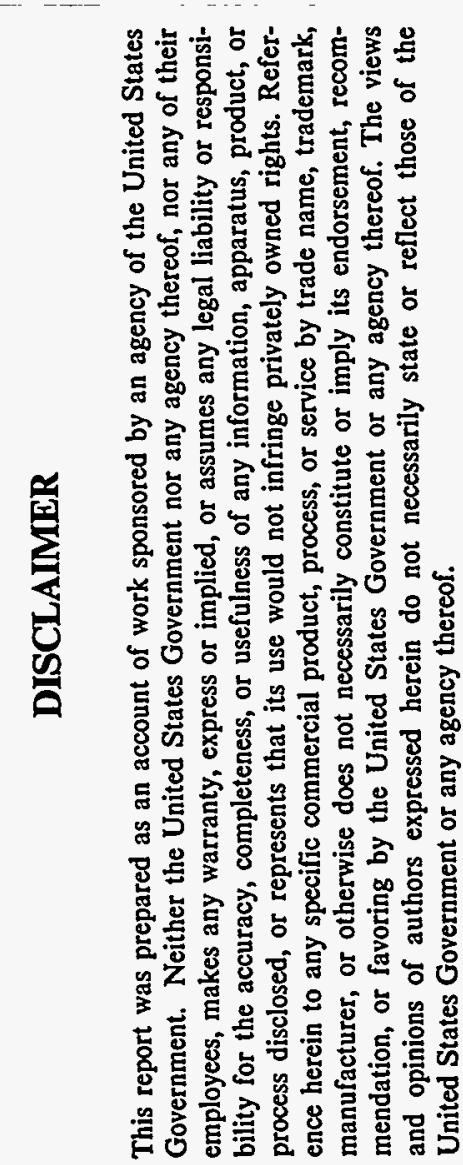

"Work supported by the U.S. Department of Energy, Energy Research Programs under Contract W-31-09-ENG-38. 


\title{
Systematic Construction Of Qualitative Physics- Based Rules For Process Diagnostics
}

\author{
Jaques Reifman \\ Argonne National Laboratory \\ Reactor Analysis Division \\ Argonne, Illinois 60439 \\ U.S.A. \\ e-mail: jreifman@anl.gov
}

\author{
Thomas Y. C. Wei \\ Argonne National Laboratory \\ Reactor Engineering Division \\ Argonne, Illinois 60439 \\ U.S.A.
}

\begin{abstract}
A novel first-principles-based expert system is proposed for on-line detection and identification of faulty component candidates during incipient off-normal process operations. The system performs function-oriented diagnostics and can be reused for diagnosing single-component failures in different processes and different plants through the provision of the appropriate process schematics information. The function-oriented and process-independent diagnostic features of the proposed expert system are achieved by constructing a knowledge base containing three distinct types of information, qualitative balance equation rules, functional classification of process components, and the process piping and instrumentation diagram. The various types of qualitative balance equation rules for processes utilizing single-phase liquids are derived and their usage is illustrated through simulation results of a realistic process in a nuclear power plant.
\end{abstract}

\subsection{Introduction}

For over a decade researchers and engineers have been developing expert systems (ESs) for process fault diagnostics. In the early systems, the knowledge base (KB) was constructed through pre-analysis of the failure of each process component based on an event-oriented approach. Once the symptoms or process signal trends associated with each component fault were identified, diagnostic rules were constructed that directly mapped symptoms into specific component faults. ${ }^{1}$ In the later systems, the $\mathrm{KB}$ was constructed by modeling the process through graph structures 2,3 where a function-oriented approach was generally used for diagnostics. Once the functions of each process component were known and the process model developed, diagnostics were accomplished by comparing the process functions that were disrupted with the components responsible for performing the disrupted functions. The advantage of this approach over event-oriented is that there is a limited number of functions that a component is designed to provide, while there are numerous possible combinations of component failures.

In spite of the progress made over the last decade, process diagnostics ESs are still limited in one fundamental aspect. They are designed to diagnose component faults for one specific process. New ESs need to be designed for different processes, unless the processes are identical, even if the processes share common properties such 
as similar thermal-hydraulic (T-H) geometrical configurations, operating conditions, and fluid material and phase.

Here, we propose a novel function-oriented first-principles-based concept for developing process diagnostic ESs that overcomes this limitation. Except for the selfcontained process schematics representation, the $\mathrm{KB}$ of the proposed system is process-independent, which allows for usage of the same diagnostic system with different processes and plants. This possibility not only decreases the effort involved in developing a new system, but it also eliminates the time-consuming process of $\mathrm{KB}$ verification and validation, which needs to be performed only once in the proposed approach. The process-independent diagnostic capability of the proposed ES is attained through the use of qualitative reasoning where a small number of values are taken to represent the values of continuous real-valued variables.

Figure 1 illustrates the three types of knowledge of the proposed diagnostic ES and their corresponding usage, in a three-step mapping, that relates process symptoms into component faults. ${ }^{4,5}$ When a process component fails, it causes the process T-H variables, e.g., pressure $P$, flow $W$, temperature $T$, and level $L$, to vary or trend from their expected values. The physical rules database (PRD) is used to map the trend in the $\mathrm{T}-\mathrm{H}$ variables into imbalance trends in the three conservation types of mass, energy, and momentum, e.g., mass increase, momentum decrease. Then, the component classification dictionary (CCD) is applied to map the identified imbalance type and trend into generic faulty component types, e.g., pump, valve, heat exchanger, whose failure could have been responsible for the identified imbalance, i.e., the inadequate performance of one of the three $\mathrm{T}-\mathrm{H}$ functions, mass transfer, energy transfer, or momentum transfer. Finally, the piping and instrumentation diagram (PID) containing the process schematics information, the only system-dependent portion of the $\mathrm{KB}$, is applied to identify specific faulty components, e.g., pump $\mathrm{A}$, valve $C V-121$, regenerative heat exchanger $C$, as the possible faulty component candidates.

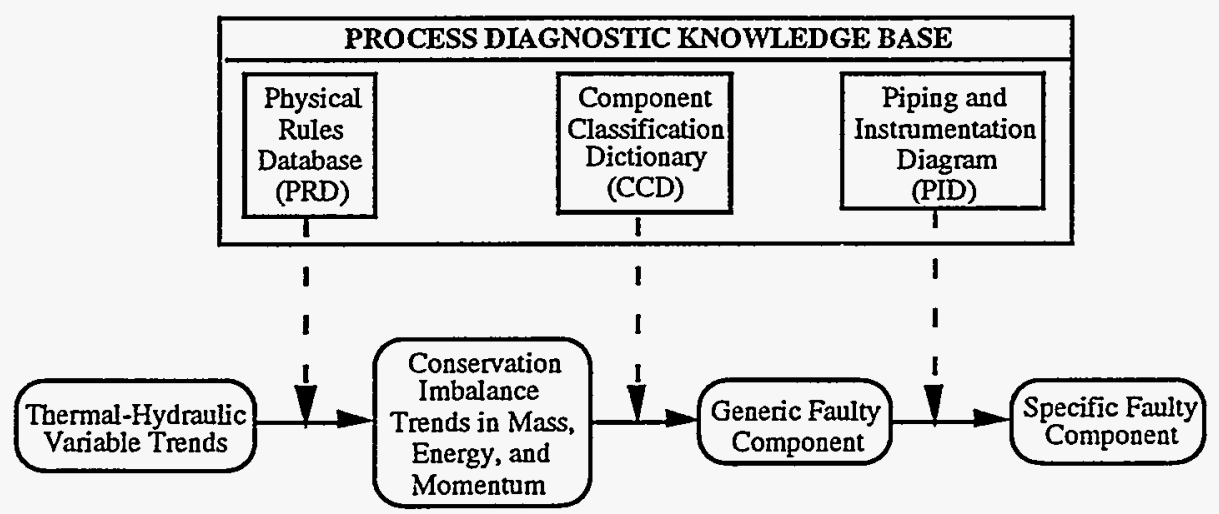

Fig. 1. Three Distinct Types of Knowledge in the Knowledge Base Perform Three Mappings Required for Process-Independent Function-Oriented Diagnostics 
In previous work reporting the proposed concept, 4,5 the first-principles qualitative rules of the PRD used to map trends in the T- $\mathrm{H}$ variables into imbalances of mass, energy, and momentum, were solely derived through the equations of state (EOS) and the definition of momentum. Furthermore, each process component was associated with one control volume that required complete instrumentation, i.e., each control volume was characterized by lumped values of $\mathrm{P}, \mathrm{W}, \mathrm{T}$, and $\mathrm{L}$, which are rarely available for each process component in real-world applications. Here, in addition to the EOS and definition of momentum, we apply the basic mathematical description of the macroscopic conservation equations to construct qualitative rules ${ }^{6}$ that characterize imbalances in mass, energy, and momentum. The locations of the existing process instruments that measure the $\mathrm{T}-\mathrm{H}$ variables define the boundaries of the control volume for each rule, which may now involve one or more connected components. That, in addition to the provision of different types of rules with different instrumentation requirements and rules capable of inferring the trend of nonmeasured variables, reduce the need for complete instrumentation. In the following sections, we derive the various types of qualitative rules of the PRD and illustrate their usage through simulation results of a process in a nuclear power plant. In this paper, the derived rules are limited to single-component failures in generic $\mathrm{T}-\mathrm{H}$ processes consisting of single-phase liquid, i.e., subcooled water. However, the rules in the PRD of the developed ES also account for separated volume components containing noncondensable gas over single-phase liquid.

\subsection{Qualitative Physics-Based Rules}

The qualitative physics rules that we derive in this section are generic rules in the sense that the same set of rules can be applied to different component types and different $\mathrm{T}-\mathrm{H}$ processes without any need for customization. This approach differs from the qualitative physics reasoning methods based on De Kleer and Brown's work, ${ }^{6}$ where a set of balance equations or confluences that model the process are customized for each device (process) based on the device topology. However, to permit the generalization of the rules, the geometrical configuration of the T-H system is decomposed into T-H loops, which are classified as being of two types, open and closed. A loop is defined as a continuous circuit of fluid flowing monotonically in one direction. A closed loop starts and ends at the same location, while an open loop starts at two different pressure boundaries. This decomposition of the $\mathrm{T}-\mathrm{H}$ system into decoupled $\mathrm{T}-\mathrm{H}$ loops allows the physical rules representing the mathematical conservation equations to be applied to any control volume configuration, i.e., one specific component or a group of connected components, within a T-H loop. The rules can not be applied to control volume configurations composed of components belonging to different T-H loops. This is due to our approach which follows a monotonically varying pressure distribution with account taken for the step discontinuity at the pump.

The qualitative physics rules of the PRD are of two classes: $Q$ rules and CV rules. A $Q$ rule indicates the type and trend of the imbalance in a control volume 
inferred from the trends in the $\mathrm{T}-\mathrm{H}$ variables. Corresponding to the three balance equations of mass, energy, and momentum, we have three types of $Q$ rules, $Q_{\text {mass, }}$, $Q_{\text {eng, and }} \mathrm{Q}_{\mathrm{mom}}$, respectively, which can bave one of three trends or qualitative values, increasing $(\uparrow)$, decreasing $(\downarrow)$, and unchanging (-). Thus, if a control volume is experiencing a loss of mass, a $Q$ rule identifying such imbalance would characterize the $\mathrm{Q}$ status of the control volume as $\mathrm{Q}_{\text {mass }}^{\downarrow}$. A CV rule infers the trend status of nonmeasured $T-H$ variables, pressure $P$, flow $W$, temperature $T$, and level $L$, in a process component, from the other $\mathrm{T}-\mathrm{H}$ variables and the $\mathrm{Q}$ status of the component. In the following paragraphs, we illustrate the derivations of the $Q$ rules and $C V$ rules.

\subsection{Derivation of $Q$ Rules}

Different classes of $Q$ rules, with varying degree of diagnostic precision, can be derived as a function of the type, trend, and number of T-H variables. Specific groups of three-signal variables with specific trends are required to form the minimum set for unique identification of an increasing or decreasing $Q$ status in a control volume. For instance, the three-signal variables [ $\left.\mathrm{P}^{\downarrow} \mathrm{W}_{\text {in }}^{\uparrow} \mathrm{W}_{\text {out }}^{\downarrow}\right]$, can uniquely identify $Q_{\text {mass }}^{\downarrow}$ in the control volume defined by the two flow measurements in both open and closed $\mathrm{T}$ $\mathrm{H}$ loop configurations. In the above notation, $\mathrm{P}^{\downarrow}$ indicates a pressure decrease measurement anywhere in the T-H loop, $W_{\text {in }}^{\uparrow}$ represents an increase in the control volume inlet flow and $W_{\text {out }}^{\downarrow}$ represents a decrease in the control volume outlet flow. Unique $Q$ status identification can also be obtained for $Q_{\operatorname{mom}}$ and $Q_{e n g}$ if the specific variable trends are available for the sets [W $\left.\mathrm{P}_{\text {in }} \mathrm{P}_{\text {out }}\right]$ and $\left[\mathrm{W} \mathrm{T}_{\text {in }} \mathrm{T}_{\text {out }}\right]$, respectively. However, in many practical situations, the instrumentation set is insufficient to provide this minimum set. There are cases where only two- or one-signal variables are available in a loop. In such cases, $Q$ rules can also be constructed to provide some malfunction $\mathrm{Q}$ diagnostics. But as can be expected, the precision of the diagnostics decreases with a larger number of possible $Q$ malfunctions being inferred. For instance, if only the two-signal variable set $[\mathrm{P} \mathrm{W}]$ is available in the loop, then a $\mathrm{Q}$ rule would indicate both $\mathrm{Q}_{\text {mass }}$ and $\mathrm{Q}_{\mathrm{mom}}$ problems.

In the following paragraphs, we derive $Q$ rules with both three- and twovariable sets. Also, we show that three-variable rules can be systematically constructed through the logical intersection of two two-variable rules, and that twovariable rules can be constructed through the logical union of two three-variable rules.

\section{Three-Variable Rules}

We start the derivation of three-variable rules for $Q$ rules that infer imbalances in the conservation of mass inventory. For the control volume shown in Fig. 2, associated with one or a group of connected components, the static mass conservation equation is given by

$$
\mathrm{Q}_{\text {mass }}=\mathrm{W}_{\text {out }}-\mathrm{W}_{\mathrm{in}}
$$

where $W_{\text {in }}$ and $W_{\text {out }}$ are the control volume inlet and outlet mass flow rates, respectively, and Qmass is the mass source/sink term in the mass balance. Reference 7 discusses how we separate the dynamic effects of a process component malfunction 
from the analysis of the static conservation equations. Transforming Eq. (1) into qualitative differential expressions using De Kleer and Brown's ${ }^{6}$ methodology and notation, gives the following confluence:

$$
\left[\mathrm{dW}_{\text {in }}\right]-\left[\mathrm{dW}_{\text {out }}\right]=-[\mathrm{dQ} \text { mass }],
$$

where the square brackets [-] represent the qualitative value or trend $(\uparrow, \downarrow,-)$, of the argument basic quantity, i.e., $W_{i n}, W_{\text {out }}$, and $Q_{\text {mass. }}$ Equation (2) represents the general confluence, from which $Q$ rules characterizing imbalances in $Q_{\text {mass }}$ can be derived by applying the different trend combinations of $\mathrm{W}_{\text {in }}$ and $\mathrm{W}_{\text {out }}$, and using the operations of qualitative algebra. ${ }^{6}$ For the case where inlet flow into the control volume is increasing and outlet flow is decreasing, the confluence in Eq. (2) infers that $\mathrm{Q}_{\text {mass }}$ is decreasing $\left(\mathrm{Q}_{\text {mass }}^{\downarrow}\right)$, or, equivalently, that the control volume is loosing mass, represented through the rule:

$$
\text { rule (A) If } W_{\text {in }}^{\uparrow} \text { and } W_{\text {out }}^{\downarrow} \text {, Then } Q_{\text {mass }}^{\downarrow} \text {. }
$$

While $\mathrm{Q}_{\mathrm{mass}}$ in the actual balance equation is the source/sink term, in the qualitative analysis rule in Eq. (3), it should be thought of as a conservation imbalance indicator, viz. a malfunction status indicator characterizing the fact that one of the components within the control volume is malfunctioning and causing the mass inventory to decrease.

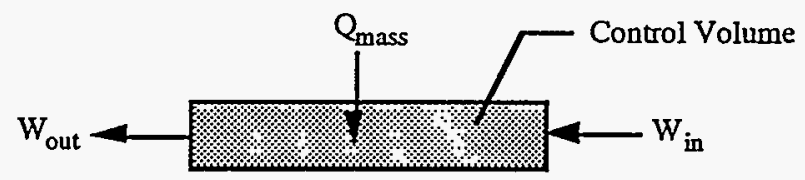

Fig. 2. Control Volume with Moveable Boundaries Defined by the Location of the Flow Measurements $\mathrm{W}_{\text {in }}$ and $\mathrm{W}_{\text {out }}$

Similarly, we can also derive a rule from the general confluence in Eq. (2) corresponding to rule (A), for the case where $Q_{\text {mass }}$ is increasing,

$$
\text { rule (B) If } \quad W_{\text {in }}^{\downarrow} \text { and } W_{\text {out }}^{\uparrow} \text {, Then } Q_{\text {mass }}^{\uparrow} \text {. }
$$

Trend combinations that cause ambiguous inference in Eq. (2), e.g., both $W_{\text {in }}$ and $W_{\text {out }}$ increasing, are not represented in the PRD. These two rules, (A) and (B), formed with two variables of the same type, i.e., $W$, in the condition part of the rule, uniquely identify $\mathrm{Q}_{\text {mass }}$ imbalances in open loops. However, in closed loops, where the definition of "in" (upstream) and "out" (downstream) has two possible combinations, both rules would be simultaneously activated regardless of the fault location and type of mass problem. 
This undesirable situation can be eliminated, by the addition of information, if a $P$ instrument measurement is available. Through perturbation analysis of the single-phase liquid equation of state $P=P(\rho, T)$, where $\rho$ is the liquid density, we obtain

$$
d P=\frac{\partial P}{\partial \rho} d \rho+\frac{\partial P}{\partial T} d T
$$

By initiating the fault diagnosis when $\mathrm{dT}$ is small and using the fact that the bulk modulus $(\rho \partial \mathrm{P} / \partial \rho)$, for liquid water is positive, the qualitative differential equation for Eq. (5) becomes

$$
[\mathrm{dP}]=[\mathrm{dM}]
$$

where $M$ is the liquid water mass inventory in a control volume $V$ with density $\rho$. Instantiating the confluence in Eq. (6) with a decreasing pressure trend, translates into the rule

$$
\text { rule (C) If } \mathrm{P}^{\downarrow} \text {, Then } \mathrm{M}^{\downarrow} \text {. }
$$

If a $\mathrm{P}$ meter is available in a closed (or open) loop, an indication of $\mathrm{P}^{\downarrow}$, and hence $M^{\downarrow}$, would contradict an inference made by rule (B). Thus, the logical combination of rule (C) with rules (A) and (B) eliminates the possibility of applying rule (B) and uniquely identifies the $\mathrm{W}^{\uparrow}$ instrument as being upstream of the malfunction and $\mathrm{W}^{\downarrow}$ instrument as being downstream of the malfunction. The logic intersection of rules $(\mathrm{A})$ and $(\mathrm{C})$ is therefore

$$
\text { rule (D) If } \mathrm{P}^{\downarrow} \text { and } \mathrm{W}_{\text {in }}^{\uparrow} \text { and } \mathrm{W}_{\text {out }}^{\downarrow} \text {, Then } \mathrm{Q}_{\text {mass }}^{\downarrow}
$$

Analogously, there is a corresponding rule for $\mathrm{Q}_{\mathrm{mom}}$ when three-signal variables, [W $\mathrm{P}_{\text {in }} \mathrm{P}_{\text {out }}$, are available,

$$
\text { rule (E) If } \mathrm{W}^{\uparrow} \text { and } \mathrm{P}_{\mathrm{in}}^{\downarrow} \text { and } \mathrm{P}_{\text {out }}^{\uparrow} \text {, Then } \mathrm{Q}_{\text {mom }}^{\uparrow} \text {, }
$$

where $Q_{\text {mom }}^{\uparrow}$ is downstream of $P_{\text {in }}$ and upstream of $P_{\text {out, }}$ and $W$ is measured anywhere in the loop. Rules (D) and $(E)$ are examples which illustrate that only three-signal variables, $\left[\mathrm{P} \mathrm{W}_{\text {in }} \mathrm{W}_{\text {out }}\right]$ or $\left[\mathrm{W} \mathrm{P}_{\text {in }} \mathrm{P}_{\text {out }}\right.$, are required to form the minimum set for unique $Q_{\text {mass }}^{\uparrow \downarrow}$ and $Q_{\text {mom }}^{\uparrow \downarrow}$ identification for the complete set of loops.

\section{Two-Variable Rules}

When only two- or one-signal variables are available in a loop, rules can also be constructed to provide some malfunction $Q$ diagnostics. But as can be expected, the precision of the diagnostics decreases with a larger number of possible $Q$ malfunctions being inferred. We provide an illustration of one such rule, for the case 
when only two-signal variables [P W], are available and show how the rule is constructed.

For the case where $\left[\mathrm{P}^{\downarrow} \mathrm{W}^{\uparrow}\right.$ ], rule (D) could be activated, if another flow meter downstream of $W$ was present with a decreasing trend, or rule $(E)$ could be activated, if another pressure meter downstream of $P$ was present with an increasing trend. Since either rule could be activated in this $\left[\mathrm{P}^{\downarrow} \mathrm{W}^{\uparrow}\right]$ combination, then the logic union of rules (D) and (E), could be applied

$$
\text { rule (F) If } \mathrm{P}^{\downarrow} \text { and } \mathrm{W}^{\uparrow} \text {, Then } \mathrm{Q}_{\text {mass }}^{\downarrow} \text { or } \mathrm{Q}_{\text {mom }}^{\uparrow} \text {, }
$$

where $Q_{\text {mass }}^{\downarrow}$ is located downstream of the $W$ instrument and $Q_{\text {mom }}^{\uparrow}$ is located downstream of the $\mathrm{P}$ instrument. Thus, when two-variable rules with different T-H variables are activated, the location of one of the variables (W for $Q_{\text {mass }}$ and $P$ for $\left.\mathrm{Q}_{\mathrm{mom}}\right)$ is used to define one boundary of the control volume with the other boundary defined by either end of the loop.

The construction of rule $(F)$ shows that there is a systematic procedure using Boolean logic, logic union in the case of two-signal variables, to derive $Q$ rules with two- or one-signal variables from the set of rules which uses the minimum threevariable sets $\left[\mathrm{P} \mathrm{W}\right.$ in $\left.\mathrm{W}_{\text {out }}\right]$ and $\left[\mathrm{W} \mathrm{P}_{\text {in }} \mathrm{P}_{\text {out }}\right]$. However, the two-variable rules can also be used to reconstruct the three-variable rules, if the signal variables can be grouped in blocks of two. For instance, if we consider a two-variable rule analogous to rule $(F)$, i.e.,

$$
\text { rule (G) If } \mathrm{P}^{\downarrow} \text { and } \mathrm{W}^{\downarrow} \text {, Then } \mathrm{Q}_{\text {mass }}^{\downarrow} \text { or } \mathrm{Q}_{\text {mom }}^{\downarrow} \text {, }
$$

and a signal set $\left[\mathrm{P}^{\downarrow} \mathrm{W}_{\mathrm{jn}}^{\uparrow} \mathrm{W}_{\text {out }}^{\downarrow}\right]$ is available, it can be grouped as two two-variable sets $\left[\mathrm{P}^{\downarrow} \mathrm{W}^{\uparrow}\right]$ and $\left[\mathrm{P}^{\downarrow} \mathrm{W}^{\downarrow}\right]$. This would mean the activation of both rules $(\mathrm{F})$ and (G), where the logical intersection of these rules is $Q_{\text {mass }}^{\downarrow}$, which is the identical conclusion of the activation of rule (D). This shows the logical consistency between the derivation of the sets of the different-variable-number rules. We apply logical union when we construct two-variable rules from two three-variable rules and logical intersection when we construct three-variable rules from two two-variable rules.

\subsection{Derivation of $\mathrm{CV}$ Rules}

$\mathrm{CV}$ rules infer the trend status of nonmeasured variables of a process component based on the trends of other $\mathrm{T}-\mathrm{H}$ variables and the $\mathrm{Q}$ status of the component. We illustrate the derivation of a $\mathrm{CV}$ rule that infers the trend of the flow $\mathrm{W}_{\mathrm{a}}$, through the cold side of a counter current heat exchanger. For the control volume shown in Fig. 3, the static energy conservation equation is given by

$$
Q_{\text {eng }}=W_{a}\left(h_{\text {out }}-h_{\text {in }}\right)=W_{a} c_{p}\left(T_{\text {out }}-T_{\text {in }}\right),
$$


where $h_{\text {in }}$ and $h_{\text {out }}$ are the control volume inlet and outlet enthalpy, respectively, $c_{p}$ is the specific heat and $Q_{\text {eng }}$ is the energy source/sink term in the energy balance. Transforming Eq. (12) into a qualitative differential expression and solving for $\mathrm{W}_{\mathrm{a}}$ yields the confluence

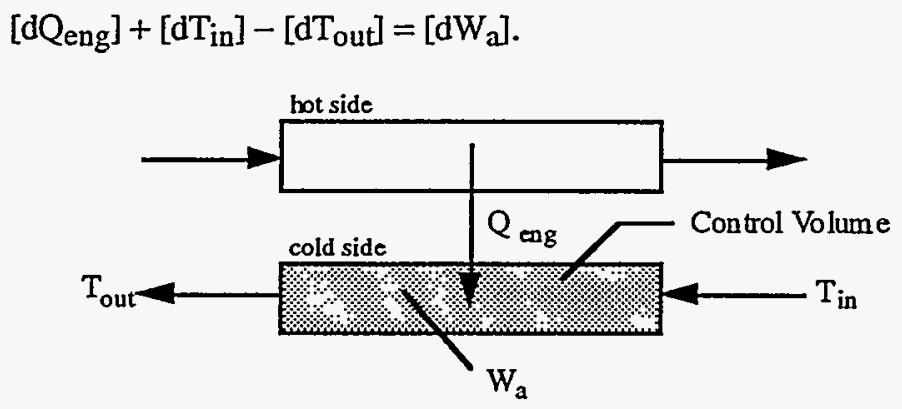

Fig. 3. Control Volume Representation of the Cold side of a Counter Current Heat Exchanger where the Energy Balance Equation Allows for the Inference of the Trend of the Flow $\mathrm{W}_{\mathrm{a}}$ Through the Heat Exchanger

For the case where the energy source into the control volume is not increasing, the inlet temperature is not increasing, and the outlet temperature is increasing, the confluence infers that the flow rate is decreasing, represented through the rule:

$$
\text { rule }(\mathrm{H}) \quad \text { If } \quad \mathrm{Q}_{\text {eng }}^{\prime \uparrow} \text { and } \mathrm{T}_{\text {in }}^{\prime \uparrow} \text { and } \mathrm{T}_{\text {out }}^{\uparrow} \text {, Then } \mathrm{W}_{\mathrm{a}}^{\downarrow} \text {, }
$$

where the symbol "/" indicates negation. Other CV rules for inference of $\mathrm{W}_{\mathrm{a}}$ can be obtained by instantiating the quantities in the left hand side of the confluence in Eq. (13) with different trend combinations. A similar procedure is used to derive CV rules for other types of T-H variables and components.

The synthetic signal trends obtained through the CV rules extract the most information possible from the process and increase the total number of instruments (virtual and real) available for diagnostics. Once inferred, synthetic signals are combined with actual signals and used to determine the $Q$ status of components through the $Q$ rules. In the following section, we illustrate the usage of $C V$ and $Q$ rules in the diagnostics of a component failure in a nuclear power plant process.

\subsection{Simulation Results}

The concepts discussed in the previous section have been incorporated into the process diagnostic system PRODIAG. The current version of PRODIAG is written in Quintus Prolog 8 and has been designed to identify single-component failures in generic $\mathrm{T}-\mathrm{H}$ processes consisting of single-phase liquid, i.e., subcooled water, and single-phase liquid plus noncondensable gas. In the following, we show simulation results of one, out of many fault events, in the Chemical and Volume Control System 
(CVCS) of a pressurized water nuclear reactor for which PRODIAG bas been used to perform diagnostics. ${ }^{9.10}$ A blind test with thirty-nine events simulated with a fullscale operator training simulator has been used to validate PRODIAG. Out of the 39 events, 37 were correctly identified within the first 40 seconds with graded degree of accuracy, i.e., uniquely identified, identified as one of two candidates, etc.

Figure 4 illustrates a portion of the letdown line and charging line of a CVCS for a pressurized water reactor. Letdown water leaves the Reactor Coolant System (RCS), indicated in the upper left portion of the figure, and flows through the shellside or hot-side of the regenerative heat exchanger $(\mathrm{RHX})$ where it gives up its heat to makeup water being returned to the RCS. From there, letdown water proceeds through a series of components (not represented in Fig. 4) until it reaches the Volume Control Tank (VCT). Then, the charging pump (pump A in the figure) takes the coolant from the VCT through the tube-side or cold-side of the RHX and back to the RCS. This simplified piping and instrumentation diagram configuration of the CVCS is decomposed into two open T-H loops, loop 1 representing the letdown line from the RCS to the VCT and loop 2 representing the charging line from the VCT to the RCS. Other flow lines, i.e., T-H loops, are represented in the PID database of PRODIAG but have been removed from the figure for simplification.

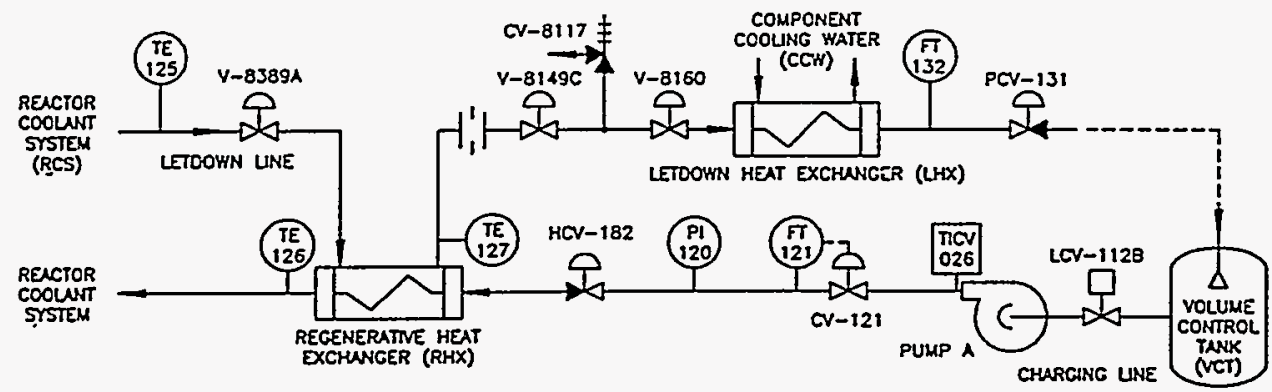

Fig. 4. Simplified Letdown Line and Charging Line of a Chemical and Volume Control System for a Pressurized Water Reactor

Let us consider the fault event where the pipe in the charging line (loop 2) connecting valve HCV-182 and the RHX starts leaking. The charging line leak causes an instantaneous decrease in loop 2 pressure, except for the separated volume VCT, measured by PI-120, increase in mass flow rate from the VCT up to the leak location measured by FT-121, and decrease in mass flow rate from the leak location to the RCS. The absence of a flow meter downstream of the leak precludes the detection of the decrease in makeup water through the tube-side (cold-side) of the RHX. A decrease in the RHX cold-side flow causes an increase in the heat exchanger outlet temperatures measured by TE-126 in loop 2 and TE-127 in loop 1. However, due to the heat exchanger thermal inertia, there is a time delay before the RHX outlet temperatures increase to reflect the pipe leak in loop 2. 
When the fault event starts, PI-120 indicates a pressure decrease and FT-121 indicates a flow increase. With only these two $\left[\mathrm{P}^{\downarrow} \mathrm{W}^{\uparrow}\right]$ measurements, the twovariable rule in the PRD, rule $(F)$, is activated by PRODIAG to hypothesize that there is either a mass decrease in loop 2 downstream of FT-121 or a momentum increase in loop 2 downstream of PI-120. The lack of an additional flow meter downstream of the leak location, precludes the differentiation between the two hypotheses. However, a few seconds into the transient, the RHX outlet temperature measured by TE-126 starts to increase, allowing PRODIAG to rule out the momentum problem and narrow down the diagnostics to a mass decrease problem.

The ES diagnostic rules of the PRD arrive at the correct diagnosis by sequentially applying CV rule $(\mathrm{H})$, and then $\mathrm{Q}$ rule $(\mathrm{D})$. First, $\mathrm{CV}$ rule $(\mathrm{H})$ is applied to infer that flow $\mathrm{W}_{\mathrm{a}}$ through the cold-side of the RHX is decreasing. With this information, $Q$ rule (D) is applied by creating a control volume from valve CV-121 to the RHX, by matching $W_{\text {in }}$ with FT-121 and $W_{\text {out }}$ with $W_{a}$, to infer that mass is decreasing.

PRODIAG applies a few other rules, not described in this paper, before CV rule $(\mathrm{H})$ can be activated. First, the enthalpy (or temperature) transport rules are applied in both loops to transport the constant temperature trend measured by TICV- 026 and TE125 to the inlet of the RHX for the cold- and hot-sides, respectively, of the heat exchanger. This procedure allows for the inference of a constant inlet temperature $T_{\text {in }}$ in both sides of the RHX and accounts for the second term $T_{i n}^{\prime} T^{\top}$, in the condition part of rule $(\mathrm{H})$. The third term in the expression $\mathrm{T}_{\text {out }}^{\uparrow}$, is directly accounted through the measured value of TE-126. Finally, the first term $Q_{\text {eng }}^{\prime \uparrow}$, is accounted for by solving an energy balance equation similar to Eq. (13) for $\mathrm{dQ}_{\text {eng }}$ in loop 1 , and reversing the obtained trend to infer $Q_{\text {eng }}^{-}$if $T E-127$ is unchanging and $Q_{\text {eng }}$ if $T E-127$ is increasing. Either case satisfies $Q_{\text {eng }}^{\prime \uparrow}$.

Once the diagnostics of the charging line leak is narrowed down to a mass decrease problem between flow meter FT-121 and the estimated flow $\mathrm{W}_{\mathrm{a}}$ inside the RHX, the ES proceeds to hypothesize the faulty component. Faulty components are hypothesized through the last two mappings illustrated in Fig. 1. First, the CCD database is searched to identify generic component types whose failure could have caused the detected mass imbalance. For mass decrease, closed valve or component break are identified. In out abstract classification of generic components, a break is classified as a sink of mass since when a component breaks or leaks it affects the mass balance causing a loss of mass. Next, the PID database is searched to determine if there are closed valves or component break between the two flow measurements. Since there are no closed valves, PRODIAG correctly hypothesizes that the faulty component is a break between FT-121 and the RHX.

\subsection{Conclusions}

In this paper we present a novel function-oriented first-principles-based ES for on-line diagnosis of incipient process component failure. Diagnostics are performed 
through a three-step mapping process, where trends in T-H variables are mapped into trends in imbalances of mass, energy, and momentum, which are then mapped into generic faulty components and next mapped into specific faulty components. The mapping from $\mathrm{T}-\mathrm{H}$ variables to imbalances in the conservation equations is performed through qualitative physics-based rules that are systematically derived in the paper. Both three- and two-variable rules are constructed, which can be used to identify imbalances in control volumes with varying diagnostics precision, depending on the type, trend, and number of instruments available. Unlike prior work describing the use of qualitative physics-based rules, here the approach is generic, with no need to customize the rules as a function of the process to be diagnosed. The proposed approach is system-independent and can be applied to different processes and plants, with the provision of the appropriate process-specific piping and instrumentation diagram.

Initial simulation results for the CVCS of a nuclear power plant indicate that the proposed ES can correctly diagnose single component failures in $\mathrm{T}-\mathrm{H}$ processes consisting of single-phase liquid plus noncondensable gas. Future work will include the testing of the ES to diagnose malfunctions in different T-H systems consisting of similar fluid properties, and extension of the current work to include T-H processes utilizing two-phase flow and multiple-component failures. Future work will also include the implementation of signal processing techniques such as low frequency bandpass filters to account for noisy signals in the determination of signal trends.

\section{Acknowledgments}

This research was supported by the U.S. Department of Energy, Energy Research Program, under contract number W-31-109-ENG-38.

\section{References}

1. W. R. Nelson, "REACTOR: An Expert System for Diagnosis and Treatment of Nuclear Reactor Accidents," Proceedings of the National Conference on Artificial Intelligence, AAAI, pp. 296-301, Pittsburgh, Pennsylvania, August 18-20, 1982.

2. F. E. Finch and M. A. Kramer, "Narrowing Diagnostic Focus Using Functional Decomposition," AIChE J., 34, 25, 1988.

3. J. E. Larsson, "Hyperfast Algorithms for Model-Based Diagnosis," Proceedings of the IEEE/IFAC Joint Symposium on Computer-Aided Control System Design," pp. 533-538, Tucson, Arizona, March 7-9, 1994.

4. J. Reifman, L. L. Briggs, and T. Y. C. Wei, "A First-Principles General Methodology for Representing the Knowledge Base of a Process Diagnosis Expert System," Proceedings of the 4th International Conference on Industrial 
and Engineering Applications of Artificial Intelligence and Expert Systems, pp. 255-265, Kauai, Hawaii, June 2-5, 1991.

5. J. Reifman, L. L. Briggs, and T. Y. C. Wei, "Nuclear Power Plant Diagnostics Using Qualitative Analysis and Component Functional Classification," Proceedings of the Frontiers in Innovative Computing for Nuclear Industry, pp. 227-236, Jackson, Wyoming, September 15-18, 1991.

6. J. De Kleer and J. S. Brown, "A Qualitative Physics Based on Confluences," AI, 24, 7, 1984.

7. J. Reifman and T. Y. C. Wei, "PRODIAG - Dynamic Qualitative Analysis for Process Fault Diagnosis," Proceedings of the 9th Power Plant Dynamics, Control and Testing Symposium, pp. 40.01-40.15, Knoxville, Tennessee, May 24-26, 1995.

8. Quintus Corporation, Palo Alto, California, Release 3, 1991.

9. J. Reifman, T. Y. C. Wei, R. G. Abboud, and T. M. Chasensky, "Cooperative Research and Development for Artificial Intelligence Based Reactor Diagnostic System," Proceedings of the American Power Conference, pp. 365-370, Chicago, Illinois, April 25-27, 1994.

10. J. Reifman, T. Y. C. Wei, R. G. Abboud, and T. M. Chasensky, "FunctionBased Approach to Plant Diagnosis," paper submitted to the American Nuclear Society 1995 Winter Meeting, San Francisco, California, October 29-November 2, 1995. 